\title{
Odontogenic Keratocyst: A Rare Presentation in Anterior Maxilla
}

\author{
Snehal Dilip Lunawat ${ }^{1}$, Varun R. Kunte ${ }^{2}$, Ajay R. Bhoosreddy ${ }^{2}$, Lalitkumar P. Gade ${ }^{3}$ and Rekha Swapnil Patil ${ }^{4}$ \\ ${ }^{1}$ Department of Oral Pathology, SMBT Institute of Dental Sciences and Research, Nashik, Maharashtra, India \\ ${ }^{2}$ Department of Oral Medicine and Radiology, MGV's KBH Dental Collage and Hospital, Nashik Maharashtra, India \\ ${ }^{3}$ Department of Oral Pathology, SMBT Dental Collage and Hospital, Sangamner, Maharashtra, India \\ ${ }^{4}$ Department of Oral Pathology, MGV's KBH Dental Collage and Hospital, Nashik Maharashtra, India
}

\begin{abstract}
The odontogenic keratocyst is an enigmatic developmental cyst that deserves special attention. It has characteristic histopathological and clinical features; but, what makes this cyst special is its aggressive behavior and high recurrence rate. Odontogenic keratocyst is a benign odontogenic cyst, commonly affecting the mandible. These lesions have posed a great difficulty for the surgeons and pathologists. Few cases affecting the maxilla are also reported. Literature search suggests that odontogenic keratocysts may affect maxillary posterior and canine region. The present case reports a lesion of odontogenic keratocyst occuring in the maxillary incisor region, crossing midline which was initially misdiagnosed as infected residual cyst. After careful evaluation, it was reported as an odontogenic keratocyst.
\end{abstract}

Key Words: Odontogenic keratocyst, Maxilla, Incisor teeth.

How to cite this article: Lunawat SD, Kunte VR, Bhoosreddy AR, Gade LP, Patil RS. Odontogenic Keratocyst: A Rare Presentation in Anterior Maxilla. J Coll Physicians Surg Pak 2020; 30(11):1226-1229.

\section{INTRODUCTION}

Philipsen coined the term 'odontogenic keratocyst' in the year 1956. ${ }^{1}$ World Health Organization classification 2005 reclassified the odontogenic keratocysts (OKCs) under benign odontogenic tumors. The new terminology given to this particular entity was keratocystic odontogenic tumor (KCOT). The evidence for reclassification was based on "aggressive growth", recurrence after treatment, the rare occurrence of a "solid" variant of OKC, and most importantly, mutations in the PTCH gene. The mutations in OKCs are not limited to PTCH, as mutations in CDKN2A, TP53, MCC, CADMI and FHIT have also been reported. PTCH gene mutations have been documented in up to $85 \%$ of syndromic (Nevoid basal cell carcinoma syndrome, NBCCS) and around 30\% of nonsyndromic OKCs. Because the NBCCS is caused by PTCH mutation, every nucleated cell in the body would contain the mutation in vertically transmitted syndromic patients. Accordingly, finding PTCH mutations in OKCs of syndromic patients is not only surprising, it is predictable.

Correspondence to: Dr. Snehal Dilip Lunawat, Department of Oral Pathology and Microbilogy, SMBT Institute of Dental Sciences and Research, Nandihills, Dhamangaon, Nashik. Maharashtra, India

E-mail: drsnehallunawat@gmail.com

Received: April 03, 2020; Revised: May 12, 2020;

Accepted: May 15, 2020

DOI: https://doi.org/10.29271/jcpsp.2020.11.1226
The roughly $15-20 \%$ of syndromic patients in whom the mutation cannotbedemonstrated, can be explained by acquiring the phenotype through somatic mutation. That leaves the justification for neoplastic pathogenesis based on the mutation in nonsyndromic patients, in which the majority of patients do not show the mutation Thus, OKCs were reincorporated in the cyst classification due to inadequate evidence. ${ }^{2}$ The etiology of OKC is probably related to the development of the dental lamina and its particular remnants. One of the characteristic features of the growth of this pathology is the tendency to grow along the cancellous channels with very little cortical expansion. The unique clinical characteristics of this entity are the local destruction and tendency for multiplicity; especially, when associated with syndromes like the NBCCS or Gorlin-Goltz syndrome. The tumour has a predilection for the mandibular third molar region and usually manifests as multilocular radiolucencies with scalloped well-defined margins. ${ }^{3}$ As per the literature search, OKC in the maxilla is a rare occurrence. However, a few cases are reported wherein maxillary canine and posterior region were involved. The present case report describes an unusual presentation of OKC in the maxillary incisor region crossing the midline.

\section{CASE REPORT}

An 80-year male patient reported to Department of Oral Medicine and Radiology, MGV's KBH Dental College and Hospital with the complaint of pain and swelling in the upper anterior teeth region for five months. Patient gave a history of extraction in the same region five months ago. The patient was well oriented to time, place and person. He did not have any medical history of underlying systemic disease. 


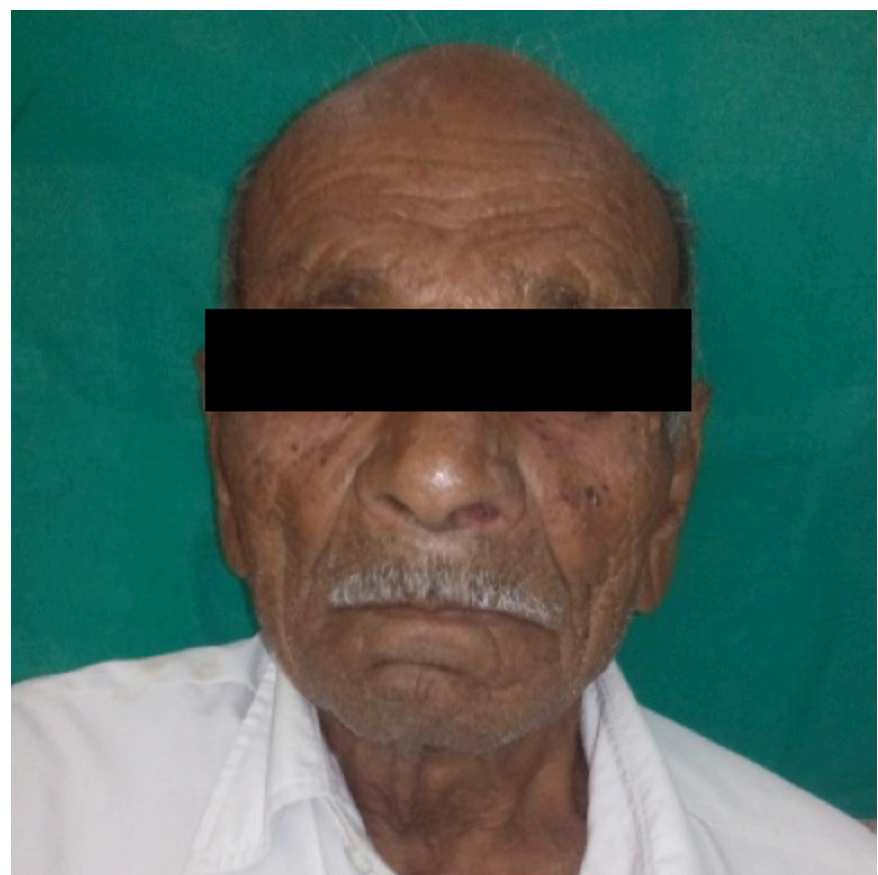

Figure 1: Extra-oral photograph of patient.

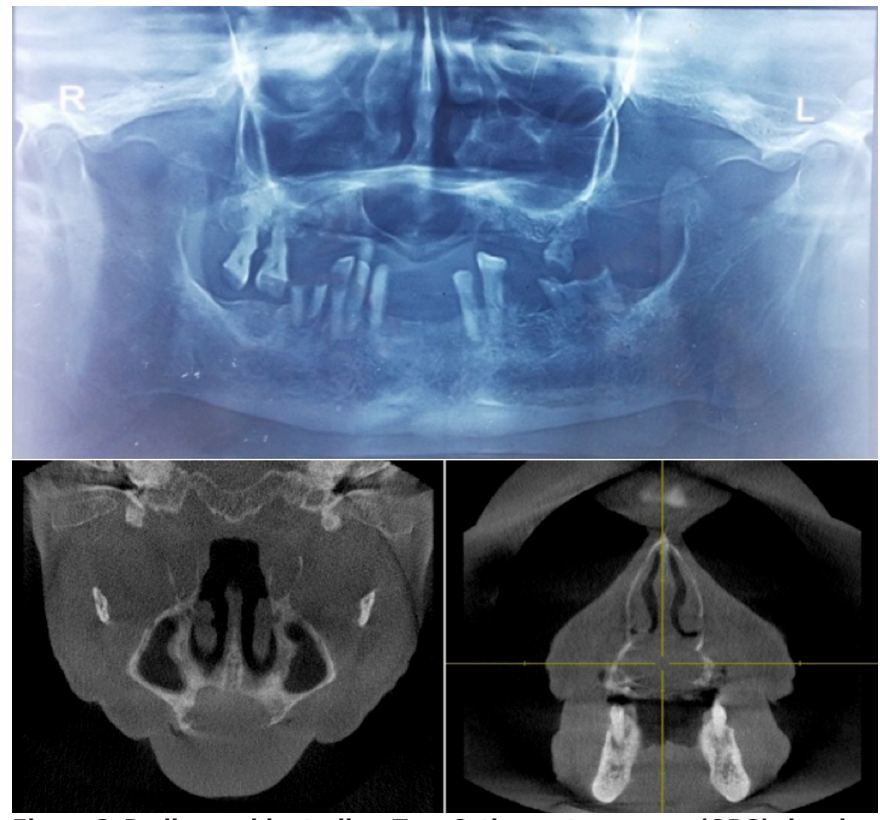

Figure 2: Radiographic studies. Top: Orthopantomagram (OPG) showing well corticated radiolucent lesion in maxillary incisor region. Bottom left: Axial cone beam computed tomography (СВCT) image. Bottom right: Coronal CBCT image.

On extraoral examination, swelling was noted above upper lip, extending $1 \mathrm{~cm}$ on each side of the philtrum (Figure 1). Intraoral examination revealed a roughly oval, $2 \times 2 \mathrm{~cm}$ swelling in the maxillary anterior region, extending $1 \mathrm{~cm}$ on either side from midline. Swelling caused obliteration of the labial vestibule with expansion of labial and palatal cortical bone. Swelling was soft to firm in consistency and perforation of labial cortical bone was suspected. Based on clinical manifestations, provisional diagnosis of "residual cyst" was given. Radiographic investigations like intraoral periapical radiograph (IOPA) showed a radiolucent lesion at the maxillary incisor region. Orthopantomagram
(OPG) revealed well corticated radiolucency in the maxillary incisor region (Figure 2). Radiolucency was approaching the floor of nasal fossa. Cone beam computed tomography (CBCT) images in cross-section and sagittal section revealed perforation of labial cortical plate of the midline radiolucency (Figure 2).

Cystic aspirate yielded yellow creamy pus. Cytological smear showed inflammatory cell infiltrate, chiefly comprising of neutrophils and lymphocytes. Few scattered red blood cells (RBCs) were also seen (Figure 3).

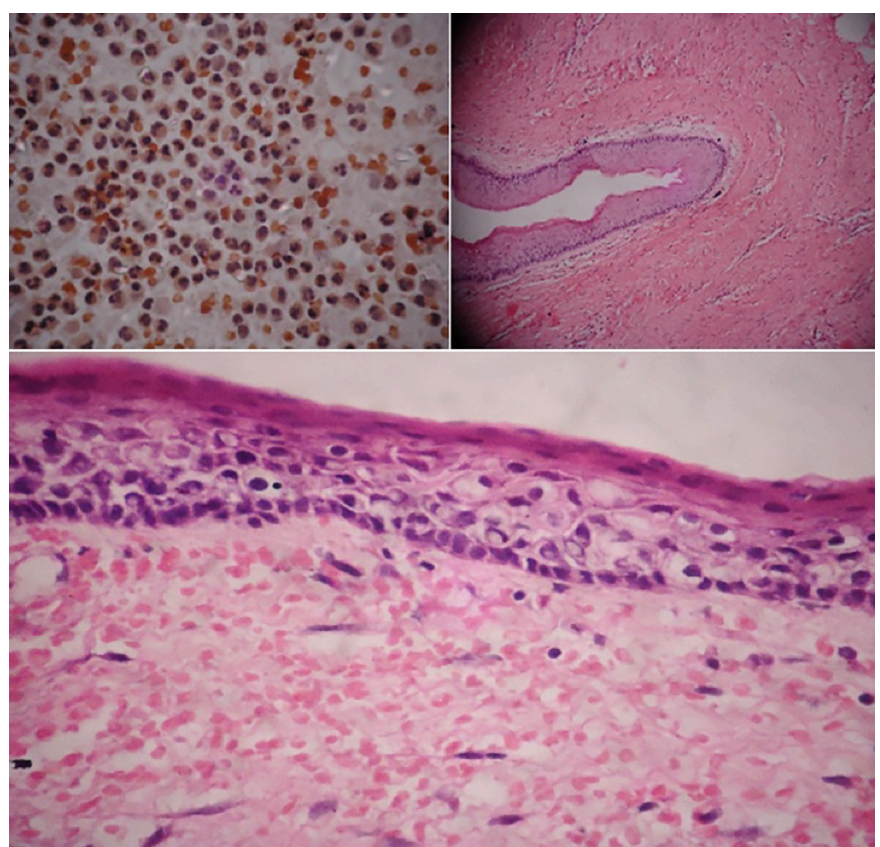

Figure 3: Top left: Aspiration cytology showing mixed inflammatory cells, chiefly neutrophils and lymphocytes and few scattered red blood cells. Top right: $\mathbf{H}$ and $\mathbf{E}$ stained section showing a cystic lumen. A parakeratinised stratified squamous epithelium with a characteristic corrugated pattern is seen lining the lumen. Bottom: High-power view showing parakeratinised stratified squamous epithelium, which is 5-7 layers thick. The basal cells show palisading with characteristic tombstone appearance.

Based on history, clinical and radiological examinations and aspiration cytology, provisional diagnosis of infected residual cyst was derived. A surgical enucleation of the cyst was planned and a written consent was taken from the patient. Post-excision, cauterisation of the tissues was done using electro-cautery. Satisfactory healing was noted.

On histopathological examination, a cystic cavity lined by stratified squamous keratinised epithelium and a supporting dense connective tissue wall were seen. The epithelium connective tissue interface was flat. The low-power magnification showed cystic lumen, lined by parakeratinised stratified squamous epithelium with a characteristic corrugated pattern. The epithelium was 5 to 7 cell thick layers, the normal thickness of epithelium found in keratocyst. At some places, the epithelium was proliferative. Diffuse inflammatory infiltrate, chiefly comprising of lymphocytes, was noted. On high-power magnification, the basal cells were showing palisading with characteristic tombstone appearance (Figure 3). Histopathological findings were suggestive of OKC. Hence, the final diagnosis was given as OKC. 
Patient has been asymptomatic for past three months. Because of the high recurrence rate OKC, periodic follow-up for the next five years has been advised to the patient.

\section{DISCUSSION}

$\mathrm{OKC}$ is one of the rare odontogenic cysts, which attracts many researchers due to its unique characteristics. OKC originates from the dental lamina remnants in the mandible and maxilla before odontogenesis is complete. It may also originate from the basal cells of overlying epithelium. It affects patients from the $1^{\text {st }}$ to $9^{\text {th }}$ decade of life with peak incidence in second to third decade and a second smaller peak is noticed in 50-70 years of age. ${ }^{4}$ It has slight male predominance. In this case, patient was in the $8^{\text {th }}$ decade. Approximately, $75 \%$ of cases of OKC affect the body of mandible, especially molar angle ramus region. Literature suggests that few cases of OKCs occur in maxilla; and there is disagreement about the most common location for OKCs in maxilla. A study showed that OKCs are divided equally between the anterior and the posterior maxillae. ${ }^{5}$ Some studies show that there are more anterior lesions than posterior. ${ }^{6}$ Still other studies conclude that the posterior region is the predominant site. According to Ali et al., canine region is the most common site of occurrence for OKCs in maxilla. ${ }^{7}$ The present case reports OKC in maxillary incisor region crossing midline, which is a rare presentation.

An important characteristic of OKC is its propensity to grow along the internal aspect of the jaws, causing minimal expansion. They are often asymptomatic and are detected on routine radiographic examination. In the present case, cortical expansion was noted and the lesion appeared to have grown labiolingually than mesiodistally.

Radiographically, OKCs have varied presentations. OKCs usually show evidence of a cortical border. The tumor may have a smooth, round or oval shape, identical to that of other cysts, or it may have a scalloped outline. The internal structure is most commonly radiolucent. The presence of internal keratin does not increase the radiopacity. In some cases, curved internal septa may be present, giving the lesion a multilocular appearance. OKCs occasionally displace and resorb teeth. $^{8}$

In the present case, CBCT was used to check the extent of the lesion. Shweel et al. stated that CBCT has great linear, volumetric and angular accuracy. ${ }^{9}$ Thus, CBCT can be used as reliable imaging tool to identify odontogenic cysts and tumors at a lesser radiation dose.

Several treatment approaches for OKCs have been suggested in the literature. Conservative approach includes simple enucleation and marsupialisation. Aggressive approach includes chemical curettage with Carnoy's solution, peripheral osteotomy, and bone resection. ${ }^{10}$ Recent advances in and determination of molecular basis of this entity, a new methodology concentrating on molecular aspects has been devised. The hedgehog $(\mathrm{Hh})$ signalling pathway can be blocked at different levels, and $\mathrm{Hh}$ inhibitors can serve as attractive antitumoragents. ${ }^{10}$

A thorough clinical and radiological evaluation is required in cases with OKC. CBCT is a reliable tool for radiographic evaluation of OKC. Histopathological examination is mandatory for every odontogenic cyst or tumor. As the OKC has high recurrence rate, adjuvant therapy in addition to aggressive enucleation is required. Misdiagnosis may lead to simple enucleation with high rate of recurrence. Periodicfollow-up isneeded to identify recurrences at the earliest.

\section{PATIENT'S CONSENT:}

Informed consent was obtained from the patient for publishing his details and data.

\section{CONFLICT OF INTEREST:}

The authors declared no conflict of interest with respect to the authorship, and/orpublication of this article.

\section{AUTHORS' CONTRIBUTION:}

SDL: Concept, design, definition and intellectual content, literature search, data acquisition, data analysis, and manuscript preparation and editing.

VK: Concept, design, definition and intellectual content.

$A B$ : Data acquisition, data analysis, and manuscript preparation and editing.

LG: Design, definition and intellectual content.

RP: Concept, design, definition and intellectual content.

\section{REFERENCES}

1. Wright JM. The odontogenic keratocyst: Orthokeratinized variant. Oral Surg Oral Med Oral Pathol 1981; 51(6):609-18. doi: 10.1016/s0030-4220(81)80011-4.

2. Wright JM, Vered M. Update from the 4th edition of the World Health Organization classification of head and neck tumours: Odontogenic and maxillofacial bone tumors. Head Neck Pathol 2017; 11(1):68-77. doi: 10.1007/s12105017-0794-1.

3. Menon S. Keratocystic odontogenic tumours: Etiology, pathogenesis and treatment revisited. J Maxillofac Oral Surg 2015; 14(3):541-7. doi: 10.1007/s12663-014-0734-5.

4. Nayak MT, Singh A, Singhvi A, Sharma R. Odontogenic keratocyst: What is in the name? J Nat Sci Biol Med 2013; 4(2):282-5. doi: 10.4103/0976-9668.116968.

5. Payne TF. An analysis of the clinical and histopathologic parameters of the odontogenic keratocyst. Oral Surg Oral Med Oral Pathol 1972; 33(4):538-46. doi.org/10.1016/ 0030-4220 (72)90366-0.

6. Panders AK, Hadders HN. Solitary keratocysts of the jaws. J Oral Surg 1969; 27(12): 931-8.

7. Ali M, Baughman R. Maxillary odontogenic keratocyst: A common and serious clinical misdiagnosis. J Am Dent Assoc 2003; 134(7):877-83.doi: 10.14219/jada.archive. 2003. 0286.

8. White S, Pharoah M. Oral radiology principles and interpretation. 7th ed. St. Louis: Elsevier 2014; 343-45.

9. Shweel M, Amer M, El-shamanhory A. A comparative study 
of cone-beam CT and multidetector $\mathrm{CT}$ in the preoperative assessment of odontogenic cysts and tumors. Egyptian J Radiol Nucl Med 2013; 44(1): 23-32. doi.org/ 10.1016/ j.ejrnm.2012.12.002.
10. Ozkan A, Bayar G, Altug H, Sencimen M, Senel B. Management of keratocystic odontogenic tumour with marsupialisation, enucleation and carnoy's solution application: A case report. Oral Health Dent Manag 2012; 11(2):69-73

\footnotetext{
$\bullet \bullet \bullet \bullet \bullet \bullet \bullet \bullet \bullet \bullet$
} 